PROCEEDINGS OF THE

AMERICAN MATHEMATICAL SOCIETY

Volume 125, Number 11, November 1997, Pages 3213-3228

S $0002-9939(97) 04186-5$

\title{
NECESSARY AND SUFFICIENT CONDITIONS FOR THE SOLVABILITY OF A PROBLEM OF HARTMAN AND WINTNER
}

\author{
N. CHERNYAVSKAYA AND L. SHUSTER
}

(Communicated by Hal L. Smith)

\begin{abstract}
The equation (1) $\left(r(x) y^{\prime}(x)\right)^{\prime}=q(x) y(x)$ is regarded as a perturbation of $(2)\left(r(x) z^{\prime}(x)\right)^{\prime}=q_{1}(x) z(x)$, where the latter is nonoscillatory at infinity. The functions $r(x), q_{1}(x)$ are assumed to be continuous real-valued, $r(x)>0$, whereas $q(x)$ is continuous complex-valued. A problem of Hartman and Wintner regarding the asymptotic integration of (1) for large $x$ by means of solutions of (2) is studied. A new statement of this problem is proposed, which is equivalent to the original one if $q(x)$ is real-valued. In the general case of $q(x)$ being complex-valued a criterion for the solvability of the HartmanWintner problem in the new formulation is obtained. The result improves upon the related theorems of Hartman and Wintner, Trench, Śimśa and some results of Chen.
\end{abstract}

\section{INTRODUCTION}

In this paper we consider differential equations

$$
\begin{array}{ll}
\left(r(x) y^{\prime}(x)\right)^{\prime}=q(x) y(x), & x \in R_{+}, \\
\left(r(x) z^{\prime}(x)\right)^{\prime}=q_{1}(x) z(x), & x \in R_{+},
\end{array}
$$

where the functions $q(x), q_{1}(x), r(x)$ are continuous, $r(x)>0, q_{1}(x)$ is real, and $q(x)$, generally speaking, is a complex-valued function for $x \in R_{+}$. We assume that (1.2) does not oscillate at infinity. It is known [3] that, in this case, there exist solutions of (1.2) $u_{1}(x)$ (the principal solution) and $v_{1}(x)$ (the non-principal solution) and a point $x_{0} \in R_{+}$such that $u_{1}(x)>0, v_{1}(x)>0$ for $x \geq x_{0}$, and the following relations are satisfied:

$$
\begin{gathered}
r(x) \cdot\left[v_{1}^{\prime}(x) u_{1}(x)-u_{1}^{\prime}(x) v_{1}(x)\right]=1, \quad x \geq x_{0}, \\
\lim _{x \rightarrow \infty} \frac{u_{1}(x)}{v_{1}(x)}=0, \int_{x_{0}}^{\infty} \frac{d t}{r(t) u_{1}^{2}(t)}=\infty, \int_{x_{0}}^{\infty} \frac{d t}{r(t) v_{1}^{2}(t)}<\infty .
\end{gathered}
$$

In recent years, the following problem has been actively investigated: find conditions under which there exists a fundamental system of solutions (FSS) $\{u(x), v(x)\}$

Received by the editors December 13, 1994.

1991 Mathematics Subject Classification. Primary 34E10.

The authors were supported by the Israel Academy of Sciences under Grants 431/95 (first author) and 505/95 (second author).

(C) 1997 American Mathematical Society 
of equation (1.1) for which:

$$
\begin{aligned}
& \lim _{x \rightarrow \infty} \frac{u(x)}{u_{1}(x)}=\lim _{x \rightarrow \infty} \frac{v(x)}{v_{1}(x)}=1, \\
& r(x)\left[\frac{u^{\prime}(x)}{u(x)}-\frac{u_{1}^{\prime}(x)}{u_{1}(x)}\right]=o\left(\frac{1}{u_{1}(x) v_{1}(x)}\right), x \rightarrow \infty, \\
& r(x)\left[\frac{v^{\prime}(x)}{v(x)}-\frac{v_{1}^{\prime}(x)}{v_{1}(x)}\right]=o\left(\frac{1}{u_{1}(x) v_{1}(x)}\right), x \rightarrow \infty .
\end{aligned}
$$

P. Hartman and A. Wintner [3], [4] were the first to study the problem and, hence, we name it after them (and denote it as problem (1.5) - (1.7)). We will say that the Hartman-Wintner problem is solvable if (1.1) has FSS $\{u(x), v(x)\}$ satisfying $(1.5)-(1.7)$.

The study, started in [3], [4], was continued in [1], [5], [7]. In particular, in [5], [7] sufficient conditions for solvability of $(1.5)-(1.7)$ were found that were essentially more subtle than those in [3], [4]. In [1] a solvability criterion for (1.5) - (1.7) was established for (1.1) with a real function $q(x)$. Below we give statements of the cited works only for problem (1.5) - (1.7). (In [1], [5], [7] some other problems similar to (1.5) - (1.7) were also studied.) First let us introduce the following assumptions and notations:

Let $f(x)$ be a continuous function on $\left[x_{0}, \infty\right)$, and

$$
F(x)=\int_{x}^{\infty} f(t) d t, \quad x \geq x_{0} .
$$

We shall write $F(x) \in \mathcal{L}\left(x_{0}\right)$ if $F(x)$ converges, at least conditionally. Denote

$$
I(x)=\int_{x}^{\infty} \Delta q(t) u_{1}(t) v_{1}(t) d t, \quad C(x)=\frac{v_{1}(x)}{u_{1}(x)} \int_{x}^{\infty} \Delta q(t) u_{1}^{2}(t) d t
$$

where $x \geq x_{0}, \Delta q(x)=q(x)-q_{1}(x)$. Let us mention an important statement:

Proposition 1 ([7]). If $I(x) \in \mathcal{L}\left(x_{0}\right)$, then $C(x) \in \mathcal{L}\left(x_{0}\right)$ and

$$
|C(x)| \leq 2 A(x), A(x)=\sup _{t \geq x}|I(t)|, x \geq x_{0} .
$$

The summary of results from [1], [3], [4], [5], [7] on the solvability of (1.5) - (1.7) is found in

Theorem 1. (A) Problem (1.5) - (1.7) is solvable if any of the following conditions holds:

1) Integral $I(x)$ converges absolutely [3], [4].

2) $\int_{x}^{\infty} \Delta q(t) u_{1}^{2}(t) d t \in \mathcal{L}\left(x_{0}\right), \int_{x_{0}}^{\infty} \frac{1}{r(t) u_{1}^{2}(t)}\left(\sup _{\xi \geq t}\left|\int_{\xi}^{\infty} \Delta q(s) u_{1}^{2}(s) d s\right|\right) d t<\infty[3],[4]$.

3) $I(x) \in \mathcal{L}\left(x_{0}\right), \int_{x_{0}}^{\infty} \frac{A(t)|I(t)| d t}{r(t) u_{1}(t) v_{1}(t)}<\infty[5]$.

4) $I(x) \in \mathcal{L}\left(x_{0}\right), \int_{x_{0}}^{\infty} \frac{A(t)|C(t)| d t}{r(t) u_{1}(t) v_{1}(t)}<\infty, \varlimsup_{x \rightarrow \infty} \frac{1}{A(x)} \int_{x}^{\infty} \frac{A(t)|C(t)| d t}{r(t) u_{1}(t) v_{1}(t)}<\mu$; the statement for $\mu<3^{-1}$ was obtained in [7] and for $\mu<1$ in [5]). 
(B) Let $q(x)$ be a real function, $I(x) \in \mathcal{L}\left(x_{0}\right)$. Problem $(1.5)-(1.7)$ is solvable if and only if

$$
\int_{x_{0}}^{\infty} \frac{C(t)^{2} d t}{r(t) u_{1}(t) v_{1}(t)}<\infty \quad[1]
$$

Let us note that in [1], instead of $I(x) \in \mathcal{L}\left(x_{0}\right)$ weaker requirements were considered as well. In $[1$, Lemma 3.3] it is shown that the conditions used in part (B) of Theorem 1 are weaker than those used in parts (A1), (A2), (A3), (A4) of Theorem 1, provided that $q(x)$ is real-valued. Therefore, the purpose of our study is to extend condition (1.10) of the solvability of (1.5) - (1.7) to the case of (1.1) with a complex-valued function $q(x)$, so that the extension would cover case (A) of Theorem 1.

To this end, we find conditions (see Theorem 2 below) under which (1.1) has FSS $\{u(x), v(x)\}$ such that relations (1.5) - (1.7) are satisfied, and, as well,

$$
\int^{\infty} r(t) u_{1}(t) v_{1}(t)\left|\frac{u^{\prime}(t)}{u(t)}-\frac{u_{1}^{\prime}(t)}{u_{1}(t)}\right|^{2} d t<\infty
$$

We call such a problem, in distinction from (1.5) - (1.7), the narrow HartmanWintner problem, and denote it by $(1.5)-(1 . *)$. Let us note from the start that problem $(1.5)-(1 . *)$ has substantial content. In particular, for (1.1) with a real function $q(x)$, problems (1.5) - (1.7) and (1.5) - (1.*) are equivalent if, for example, $I(x) \in \mathcal{L}\left(x_{0}\right)$ (see section 2, Corollary 2.3 to Lemma 2.1). In a general case of (1.1) with a complex function $q(x)$, solution of problem (1.5) - (1.*) allows the weakening of conditions (A) of Theorem 1.

To state the results (Theorem 2 and Corollaries) let us introduce the functions on $\left[x_{0}, \infty\right)$ :

$$
\begin{aligned}
J(x) & =\int_{x}^{\infty} \frac{\operatorname{Re}(I(t) \overline{C(t)})}{r(t) u_{1}(t) v_{1}(t)} d t, \\
P(x) & =\int_{x}^{\infty} \operatorname{Re}(\Delta q(t) \bar{C}(t)) u_{1}(t) v_{1}(t) d t, \\
G(x) & =\int_{x}^{\infty} \frac{|C(t)|^{2} d t}{r(t) u_{1}(t) v_{1}(t)},
\end{aligned}
$$

where $J(x)$ and $P(x)$ may converge conditionally. Let us mention

Proposition 2 (See section 3, Lemma 3.1). If $I(x) \in \mathcal{L}\left(x_{0}\right)$, then all integrals (1.11) converge and diverge simultaneously.

Our main result is contained in

Theorem 2. Problem (1.5) - (1.*) is solvable if and only if $I(x) \in \mathcal{L}\left(x_{0}\right)$ and any of the integrals (1.11) converges.

Corollary. Let $I(x) \in \mathcal{L}\left(x_{0}\right)$. Then problem $(1.5)-(1 . *)$ is solvable if at least one of the conditions (I) - (III) is fulfilled:
(I) $\int_{x_{0}}^{\infty}|\Delta q(t) C(t)| u_{1}(t) v_{1}(t) d t<\infty$;
(II) $\int_{x_{0}}^{\infty} \frac{|I(t) C(t)|}{r(t) u_{1}(t) v_{1}(t)} d t<\infty$; 


$$
\text { (III) } \int_{x_{0}}^{\infty} \frac{|I(t)|^{2} d t}{r(t) u_{1}(t) v_{1}(t)} d t<\infty .
$$

In particular, using Proposition 1 we obtain that condition (I) strengthens Theorem 1 in part (A1), while condition (II) implies Theorem 1 in parts (A3), (A4) and (A2) (in [7, p. 427], it is proved that assumptions (A2) of Theorem 1 imply $\left.I(x) \in \mathcal{L}\left(x_{0}\right)\right)$. Finally, Theorem 2 itself extends part (B) of Theorem 1 to the case of complex-valued $q(x)$.

The following theorem establishes a relationship between problems $(1.5)-(1.7)$ and $(1.5)-(1 . *)$, and contains a necessary condition of solvability of $(1.5)-(1.7)$.

Theorem 3. If problem (1.5) - (1.7) is solvable, then $C(x) \in \mathcal{L}\left(x_{0}\right)$ and $C(x) \rightarrow 0$ as $x \rightarrow \infty$. Moreover, condition (1.*) follows from $(1.5)-(1.7)$ if and only if $G\left(x_{0}\right)<\infty$.

Thus, the class of equations (1.1) for which problem (1.5) - (1.7) is solvable, but problem $(1.5)-(1 . *)$ is not solvable, is not wider than the class of equations (1.1) for which $C(x) \in \mathcal{L}\left(x_{0}\right), \quad C(x) \rightarrow 0$ as $x \rightarrow \infty$ and $G\left(x_{0}\right)=\infty$.

\section{Problems equivalent to the Hartman-Wintner problem}

While studying problems $(1.5)-(1.7)$ and $(1.5)-(1 . *)$, we have as a basis the following statements:

Lemma 2.1. Problem (1.5)-(1.7) is solvable if and only if the differential equation

$$
\left(r(x) u_{1}^{2}(x) \beta^{\prime}(x)\right)^{\prime}=\Delta q(x) u_{1}^{2}(x) \beta(x), x \geq x_{0},
$$

has a solution $\beta(x)$ such that

$$
\lim _{x \rightarrow \infty} \beta(x)=1, \quad \lim _{x \rightarrow \infty} r(x) u_{1}(x) v_{1}(x) \beta^{\prime}(x)=0 .
$$

Proof. Necessity. Let problem (1.5) - (1.7) be fulfilled for FSS $\{u(x), v(x)\}$ and $\left\{u_{1}(x), v_{1}(x)\right\}$ of equations (1.1) and (1.2). Denote $\beta(x)=u(x) \cdot u_{1}(x)^{-1}, x \geq$ $x_{0}$. According to (1.5), the first equality in (2.2) is fulfilled, and (1.5) - (1.6) imply the second one:

$$
\begin{aligned}
& \lim _{x \rightarrow \infty} r(x) u_{1}(x) v_{1}(x) \beta^{\prime}(x)=\lim _{x \rightarrow \infty} r(x) \frac{v_{1}(x)}{u_{1}(x)}\left[u^{\prime}(x) u_{1}(x)-u_{1}^{\prime}(x) u(x)\right] \\
= & \lim _{x \rightarrow \infty} r(x) v_{1}(x) u(x)\left[\frac{u^{\prime}(x)}{u(x)}-\frac{u_{1}^{\prime}(x)}{u_{1}(x)}\right]=\lim _{x \rightarrow \infty} \beta(x) u_{1}(x) v_{1}(x) o\left(\frac{1}{u_{1}(x) v_{1}(x)}\right)=0 .
\end{aligned}
$$

Furthermore, it follows from (1.1) and (1.2) that

$$
\begin{aligned}
\Delta q(x) u_{1}^{2}(x) \beta(x) & =\Delta q(x) u(x) u_{1}(x)=\left(r(x) u^{\prime}(x)\right)^{\prime} u_{1}(x)-\left(r(x) u_{1}^{\prime}(x)\right)^{\prime} u(x) \\
& =\left[r(x) u^{\prime}(x) u_{1}(x)-r(x) u_{1}^{\prime}(x) u(x)\right]^{\prime}=\left[r(x) u_{1}^{2}(x)\left(\frac{u(x)}{u_{1}(x)}\right)^{\prime}\right]^{\prime} \\
& =\left(r(x) u_{1}^{2}(x) \beta^{\prime}(x)\right)^{\prime} .
\end{aligned}
$$

Sufficiency. Let $(2.1)-(2.2)$ have a solution $\beta(x)$. According to (2.2) there exists $x_{1} \geq x_{0}$ such that $|\beta(x)| \geq \varepsilon>0$ for $x \geq x_{1}$. Let us set $u(x)=\beta(x) u_{1}(x), x \geq x_{1}$, and check that $u(x)$ is a solution of (1.1). Indeed, in new notations, (2.1) takes on the form:

$$
\left[r(x) u^{\prime}(x) u_{1}(x)-r(x) u_{1}^{\prime}(x) u(x)\right]^{\prime}=\Delta q(x) u(x) u_{1}(x), x \geq x_{1} .
$$


Since

$$
\begin{aligned}
r(x) u^{\prime}(x) & =r(x)\left[\beta^{\prime}(x) u_{1}(x)+u_{1}^{\prime}(x) \beta(x)\right] \\
& =\frac{r(x) u_{1}^{2}(x) \beta^{\prime}(x)}{u_{1}(x)}+\left(r(x) u_{1}^{\prime}(x)\right) \beta(x), x \geq x_{1},
\end{aligned}
$$

then $r(x) u^{\prime}(x)$ has the dervative at $x \geq x_{1}$. Hence,

$$
\left(r(x) u^{\prime}(x)\right)^{\prime} u_{1}(x)-\left(r(x) u_{1}^{\prime}(x)\right)^{\prime} u(x)=\left(q(x)-q_{1}(x)\right) u(x) u_{1}(x), x \geq x_{1} .
$$

The latter equality and (1.2) imply that $u(x)$ is a solution of (1.1). Let

$$
v(x)=u(x) \int_{x_{1}}^{x} \frac{d t}{r(t) u^{2}(t)}, \quad x \geq x_{1} .
$$

A direct calculation shows that $v(x)$ is also a solution of (1.1) and

$$
r(x) \cdot\left[v^{\prime}(x) u(x)-u^{\prime}(x) v(x)\right]=1, x \geq x_{1} .
$$

Therefore, $\{u(x), v(x)\}$ is an FSS of (1.1). It follows from (1.3) that

$$
v_{1}(x)=c_{1} u_{1}(x)+u_{1}(x) \int_{x_{1}}^{x} \frac{d t}{r(t) u_{1}^{2}(t)}, c_{1}=\frac{v_{1}\left(x_{1}\right)}{u_{1}\left(x_{1}\right)}, x \geq x_{1} \geq x_{0} .
$$

Using the definition of $u(x),(2.2)$ and (1.4), we find

$$
\int_{x_{1}}^{\infty} \frac{d t}{r(t) u^{2}(t)}=\infty
$$

(2.2), (2.4), (1.4), (2.7) and the l'Hospital rule imply (1.5) for $v(x)$ :

$$
\lim _{x \rightarrow \infty} \frac{v(x)}{v_{1}(x)}=\lim _{x \rightarrow \infty} \frac{u(x)}{u_{1}(x)} \frac{\int_{x_{1}}^{x} \frac{d t}{r(t) u^{2}(t)}}{c_{1}+\int_{x_{1}}^{x} \frac{d t}{r(t) u_{1}^{2}(t)}}=\lim _{x \rightarrow \infty} \beta(x) \lim _{x \rightarrow \infty} \frac{1}{\beta^{2}(x)}=1 .
$$

Let us check (1.6). It follows from the definition of $u(x)$ and (2.2) that

$$
\begin{aligned}
r(x)\left[\frac{u^{\prime}(x)}{u(x)}-\frac{u_{1}^{\prime}(x)}{u_{1}(x)}\right] & =r(x) \frac{\beta^{\prime}(x)}{\beta(x)}=\frac{r(x) u_{1}(x) v_{1}(x) \beta^{\prime}(x)}{u_{1}(x) v_{1}(x)} \frac{1}{\beta(x)} \\
& =o\left(\frac{1}{u_{1}(x) v_{1}(x)}\right)
\end{aligned}
$$

as $x \rightarrow \infty$. It remains to justify (1.7). From (2.5) and (1.3) we obtain

$$
\begin{aligned}
\frac{v^{\prime}(x)}{v(x)} & =\frac{u^{\prime}(x)}{u(x)}+\frac{v_{1}(x) u_{1}(x)}{v(x) u(x)}\left[\frac{v_{1}^{\prime}(x)}{v_{1}(x)}-\frac{u_{1}^{\prime}(x)}{u_{1}(x)}\right] \\
& =\frac{v_{1}^{\prime}(x)}{v_{1}(x)}+\left[\frac{u_{1}(x) v_{1}(x)}{u(x) v(x)}-1\right]\left[\frac{v_{1}^{\prime}(x)}{v_{1}(x)}-\frac{u_{1}^{\prime}(x)}{u_{1}(x)}\right]+\left[\frac{u^{\prime}(x)}{u(x)}-\frac{u_{1}^{\prime}(x)}{u_{1}(x)}\right] \\
& =\frac{v_{1}^{\prime}(x)}{v_{1}(x)}+\left[\frac{u_{1}(x) v_{1}(x)}{u(x) v(x)}-1\right] \frac{1}{r(x) u_{1}(x) v_{1}(x)}+o\left(\frac{1}{r(x) u_{1}(x) v_{1}(x)}\right) \\
& =\frac{v_{1}^{\prime}(x)}{v_{1}(x)}+\frac{1}{r(x)} o\left(\frac{1}{u_{1}(x) v_{1}(x)}\right), \quad \text { as } \quad x \rightarrow \infty .
\end{aligned}
$$

In the following, some corollaries of Lemma 2.1 are of importance. 
Corollary 2.1. Problem (1.5) - (1.7) is solvable if and only if the integro-differential equation

$$
r(x) \beta^{\prime}(x)=-\frac{1}{u_{1}^{2}(x)} \int_{x}^{\infty} \Delta q(t) u_{1}^{2}(t) \beta(t) d t, \quad x \geq x_{0},
$$

has a solution $\beta(x)$ such that

$$
\lim _{x \rightarrow \infty} \beta(x)=1, \quad \lim _{x \rightarrow \infty} r(x) u_{1}(x) v_{1}(x) \beta^{\prime}(x)=0 .
$$

Proof. Necessity. If problem (1.5) - (1.7) is solvable, then there exists a solution of problem (2.1) - (2.2). According to (2.2) and (1.4) we have:

$$
\lim _{x \rightarrow \infty} r(x) u_{1}^{2}(x) \beta^{\prime}(x)=\lim _{x \rightarrow \infty} r(x) u_{1}(x) v_{1}(x) \beta^{\prime}(x) \frac{u_{1}(x)}{v_{1}(x)}=0 .
$$

It remains to integrate (2.1) over $[x, \infty), x \geq x_{0}$ and to use (2.10).

Sufficiency. Relations (2.1) - (2.2) follow directly from (2.8) - (2.9).

Remark 2.1. It is evident that solutions of (2.1) - (2.2) and (2.8) - (2.9) coincide.

Corollary 2.2. Problem (1.5) - (1.*) is solvable if and only if problems (2.8)(2.9), (2.1) - (2.2) have a solution $\beta(x)$ such that

$$
\int^{\infty} r(x) u_{1}(x) v_{1}(x)\left|\beta^{\prime}(x)\right|^{2} d x<\infty .
$$

Remark 2.2. Here and in the following, problems $(2.8)-(2.9)-(2 . *),(2.1)-(2.2)$ $-(2 . *)$ are denoted by $(2.8)-(2 . *),(2.1)-(2 *)$. The letter $\tau$ denotes absolute positive constants, which may be different within one proof.

Proof. Necessity. Let (1.5) - (1.*) be solvable. Then problem (2.1) - (2.2) has a solution $\beta(x)=u(x) u_{1}(x)^{-1}, x \geq x_{1}$ and $0<\varepsilon \leq|\beta(x)| \leq \tau, x \geq x_{1}$. Since

$$
\left|\beta^{\prime}(x)\right|=|\beta(x)|\left|\frac{u^{\prime}(x)}{u(x)}-\frac{u_{1}^{\prime}(x)}{u_{1}(x)}\right| \leq \tau\left|\frac{u^{\prime}(x)}{u(x)}-\frac{u_{1}^{\prime}(x)}{u_{1}(x)}\right|, \quad x \geq x_{1}
$$

then

$$
\int_{x_{1}}^{\infty} r(x) u_{1}(x) v_{1}(x)\left|\beta^{\prime}(x)\right|^{2} d x \leq \tau \int_{x_{1}}^{\infty} r(x) u_{1}(x) v_{1}(x)\left|\frac{u^{\prime}(x)}{u(x)}-\frac{u_{1}^{\prime}(x)}{u_{1}(x)}\right|^{2} d x<\infty .
$$

Sufficiency. Let $\beta(x)$ be a solution of $(2.1)-(2 . *)$. Repeating the proof of sufficiency in Lemma 2.1, we obtain that $u(x)=\beta(x) u_{1}(x)$ is a solution of (1.1), conditions (1.5) - (1.7) are fulfilled and $\inf _{x \geq x_{1}}|\beta(x)| \geq \varepsilon>0$. Then:

$$
\begin{aligned}
\int_{x_{1}}^{\infty} r(x) u_{1}(x) v_{1}(x) & \left|\frac{u^{\prime}(x)}{u(x)}-\frac{u_{1}^{\prime}(x)}{u_{1}(x)}\right|^{2} d x=\int_{x_{1}}^{\infty} r(x) u_{1}(x) v_{1}(x)\left|\beta^{\prime}(x)\right|^{2} \frac{d x}{|\beta(x)|^{2}} \\
& \leq \frac{1}{\varepsilon^{2}} \int_{x_{1}}^{\infty} r(x) u_{1}(x) v_{1}(x)\left|\beta^{\prime}(x)\right|^{2} d x<\infty .
\end{aligned}
$$

Remark 2.3. Problems (1.5) - (1.7), (1.5) - (1.*) are also equivalent to certain problems of solvability of some integral equation (see [2]).

Corollary 2.3. Let $q(x)$ be a real function and $I(x) \in \mathcal{L}\left(x_{0}\right)$. Then problems $(1.5)-(1.7)$ and $(1.5)-(1 . *)$ are equivalent.

It is convenient for us to prove this Corollary at the end of section 4. 


\section{Auxiliary Results}

We need the following lemmas (for notations see (1.8), (1.11)).

Lemma 3.1. Let $I(x) \in \mathcal{L}\left(x_{0}\right)$. Then $C(x) \in \mathcal{L}\left(x_{0}\right)$ and $H(x) \in \mathcal{L}\left(x_{0}\right)$, where

$$
H(x)=\int_{x}^{\infty} \frac{C(t) d t}{r(t) u_{1}(t) v_{1}(t)}, \quad x \geq x_{0},
$$

and the relations

$$
|C(x)| \leq 2 A(x),|H(x)|=|I(x)-C(x)| \leq A(x), A(x)=\sup _{t \geq x}|I(t)|, x \geq x_{0},
$$

are fulfilled. Furthermore, $J(x) \in \mathcal{L}\left(x_{0}\right), P(x) \in \mathcal{L}\left(x_{0}\right)$ if and only if $G\left(x_{0}\right)<\infty$. In the latter case the inequalities

$$
\begin{gathered}
|J(x)-G(x)|=\frac{1}{2}|H(x)|^{2} \leq \frac{1}{2} A^{2}(x), \quad x \geq x_{0}, \\
|P(x)-G(x)| \leq \frac{|C(x)|^{2}}{2} \leq 2 A^{2}(x), \quad x \geq x_{0},
\end{gathered}
$$

are valid. Finally, $G\left(x_{0}\right) \leq \tilde{I}\left(x_{0}\right)$, if

$$
\tilde{I}\left(x_{0}\right) \stackrel{\text { def }}{=} \int_{x_{0}}^{\infty} \frac{|I(t)|^{2} d t}{r(t) u_{1}(t) v_{1}(t)}<\infty .
$$

Proof. It follows from (1.3) - (1.4) that

$$
u_{1}(x)=v_{1}(x) \int_{x}^{\infty} \frac{d t}{r(t) v_{1}^{2}(t)}, \quad\left(\frac{u_{1}(x)}{v_{1}(x)}\right)^{\prime}<0, \quad x \geq x_{0} .
$$

The following transformation of $C(x)$ is based on integration by parts and (1.4):

$$
\begin{aligned}
C(x) & =\frac{v_{1}(x)}{u_{1}(x)} \int_{x}^{\infty} \Delta q(t) u_{1}(t) v_{1}(t) \frac{u_{1}(t)}{v_{1}(t)} d t \\
& =\frac{v_{1}(x)}{u_{1}(x)}\left[-\left.I(t) \frac{u_{1}(t)}{v_{1}(t)}\right|_{x} ^{\infty}+\int_{x}^{\infty} I(t)\left(\frac{u_{1}(t)}{v_{1}(t)}\right)^{\prime} d t\right] \\
& =I(x)-\frac{v_{1}(x)}{u_{1}(x)} \int_{x}^{\infty} \frac{I(t) d t}{r(t) v_{1}^{2}(t)} .
\end{aligned}
$$

From (3.6) and (3.5) we obtain:

$$
|C(x)| \leq|I(x)|+\frac{v_{1}(x)}{u_{1}(x)} \int_{x}^{\infty} \frac{|I(t)| d t}{r(t) v_{1}^{2}(t)} \leq|I(x)|+A(x) \leq 2 A(x) .
$$

Differentiation of $C(x)$ yields:

$$
\begin{aligned}
C^{\prime}(x) & =-\Delta q(x) u_{1}(x) v_{1}(x)+\frac{1}{r(x) u_{1}^{2}(x)} \int_{x}^{\infty} \Delta q(t) u_{1}^{2}(t) d t \\
& =-\Delta q(x) u_{1}(x) v_{1}(x)+\frac{C(x)}{r(x) u_{1}(x) v_{1}(x)} .
\end{aligned}
$$

After integrating (3.7) and using (3.1) and (3.6), we obtain:

$$
H(x)=I(x)-C(x)=\frac{v_{1}(x)}{u_{1}(x)} \int_{x}^{\infty} \frac{I(t) d t}{r(t) v_{1}^{2}(t)}, \quad x \geq x_{0} .
$$


It follows from (3.8) that $H(x) \in \mathcal{L}\left(x_{0}\right)$. Furthermore, using (3.5) and (3.8), we have

$$
|H(x)| \leq \frac{v_{1}(x)}{u_{1}(x)} \int_{x}^{\infty} \frac{|I(t)| d t}{r(t) v_{1}^{2}(t)} \leq A(x) \frac{1}{u_{1}(x)}\left[v_{1}(x) \int_{x}^{\infty} \frac{d t}{r(t) v_{1}^{2}(t)}\right]=A(x) .
$$

The first equality (3.8) implies

$$
C(x)=I(x)-\int_{x}^{\infty} \frac{C(t) d t}{r(t) u_{1}(t) v_{1}(t)}, \quad x \geq x_{0}
$$

Therefore,

$$
\begin{aligned}
\frac{|C(x)|^{2}}{r(x) u_{1}(x) v_{1}(x)} & =\frac{\operatorname{Re}(I(x) \bar{C}(x))}{r(x) u_{1}(x) v_{1}(x)}-\frac{\operatorname{Re} C(x)}{r(x) u_{1}(x) v_{1}(x)} \int_{x}^{\infty} \frac{\operatorname{Re} C(t) d t}{r(t) u_{1}(t) v_{1}(t)} \\
& -\frac{\operatorname{Im} C(x)}{r(x) u_{1}(x) v_{1}(x)} \int_{x}^{\infty} \frac{\operatorname{Im} C(t) d t}{r(t) u_{1}(t) v_{1}(t)} .
\end{aligned}
$$

Let us integrate the latter equality over $[x, \infty)$ :

$$
\begin{aligned}
G(x)= & \int_{x}^{\infty} \frac{\operatorname{Re}(I(t) \bar{C}(t)) d t}{r(t) u_{1}(t) v_{1}(t)}+\frac{1}{2}\left(\int_{x}^{\infty} \frac{\operatorname{Re} C(t) d t}{r(t) u_{1}(t) v_{1}(t)}\right)^{2} \\
& +\frac{1}{2}\left(\int_{x}^{\infty} \frac{\operatorname{Im} C(t) d t}{r(t) u_{1}(t) v_{1}(t)}\right)^{2} \\
= & J(x)+\frac{1}{2}|H(x)|^{2}, \quad x \geq x_{0} .
\end{aligned}
$$

Since $H(x) \in \mathcal{L}\left(x_{0}\right)$, then $J(x) \in \mathcal{L}\left(x_{0}\right)$ if and only if $G\left(x_{0}\right)<\infty$. Furthermore, from (3.10) and (3.1) we obtain (3.2). Also, from (3.7) we find:

$$
\operatorname{Re} C^{\prime}(x) \bar{C}(x)=-\operatorname{Re}(\Delta q(x) \bar{C}(x)) u_{1}(x) v_{1}(x)+\frac{|C(x)|^{2}}{r(x) u_{1}(x) v_{1}(x)}, \quad x \geq x_{0} .
$$

After integrating (3.11) over $[x, \infty)$ we again use (3.1) and obtain:

$$
P(x)=\frac{1}{2}|C(x)|^{2}+G(x), \quad x \geq x_{0} .
$$

Now (3.3) follows from (3.12) and (3.1). Furthermore, it follows from (3.9) that:

$\operatorname{Re} I(x)=\operatorname{Re} C(x)+\int_{x}^{\infty} \frac{\operatorname{Re} C(t) d t}{r(t) u_{1}(t) v_{1}(t)}, \operatorname{Im} I(x)=\operatorname{Im} C(x)+\int_{x}^{\infty} \frac{\operatorname{Im} C(t) d t}{r(t) u_{1}(t) v_{1}(t)}$.

Hence,

$$
\begin{aligned}
\frac{(\operatorname{Re} I(x))^{2}}{r(x) u_{1}(x) v_{1}(x)} & =\frac{(\operatorname{Re} C(x))^{2}}{r(x) u_{1}(x) v_{1}(x)}+\frac{2 \operatorname{Re} C(x)}{r(x) u_{1}(x) v_{1}(x)} \int_{x}^{\infty} \frac{\operatorname{Re} C(t) d t}{r(t) u_{1}(t) v_{1}(t)} \\
& +\frac{1}{r(x) u_{1}(x) v_{1}(x)}\left(\int_{x}^{\infty} \frac{\operatorname{Re} C(t) d t}{r(t) u_{1}(t) v_{1}(t)}\right)^{2} \\
\frac{(\operatorname{Im} I(x))^{2}}{r(x) u_{1}(x) v_{1}(x)} & =\frac{(\operatorname{Im} C(x))^{2}}{r(x) u_{1}(x) v_{1}(x)}+\frac{2 \operatorname{Im} C(x)}{r(x) u_{1}(x) v_{1}(x)} \int_{x}^{\infty} \frac{\operatorname{Im} C(t) d t}{r(t) u_{1}(t) v_{1}(t)} \\
& +\frac{1}{r(x) u_{1}(x) v_{1}(x)}\left(\int_{x}^{\infty} \frac{\operatorname{Im} C(t) d t}{r(t) u_{1}(t) v_{1}(t)}\right)^{2}
\end{aligned}
$$


These equalities imply

$$
\tilde{I}(x)=G(x)+|H(x)|^{2}+\int_{x}^{\infty} \frac{|H(t)|^{2} d t}{r(t) u_{1}(t) v_{1}(t)}, x \geq x_{0} .
$$

According to (3.13), if $\tilde{I}\left(x_{0}\right)<\infty$, then $G\left(x_{0}\right) \leq \tilde{I}\left(x_{0}\right)<\infty$.

Remark 3.1. We included Proposition 1 (section 1) in Lemma 3.1 for the sake of completeness of exposition. Proposition 2 (section 1) is contained in Lemma 3.1.

Denote $R_{b}=[b, \infty), C\left(R_{b}\right)=C[b, \infty)$ and consider the integral operator

$$
(K f)(x)=\frac{v_{1}(x)}{u_{1}(x)} \int_{x}^{\infty} \frac{C(t)}{r(t) v_{1}^{2}(t)} f(t) d t, f(t) \in C\left(R_{b}\right), b \geq x_{0} .
$$

Lemma 3.2. The following inequality holds:

$$
\|K\|_{C\left(R_{b}\right) \rightarrow C\left(R_{b}\right)} \leq \sup _{t \geq b}|C(t)|, b \geq x_{0} .
$$

Proof. The Lemma follows from (3.5):

$$
\begin{aligned}
\|K f\|_{C\left(R_{b}\right)} & \leq\left(\sup _{t \geq b}|C(t)|\right) \sup _{x \geq b} \frac{1}{u_{1}(x)}\left[v_{1}(x) \int_{x}^{\infty} \frac{d t}{r(t) v_{1}^{2}(t)}\right] \sup _{t \geq b}|f(t)| \\
& =\sup _{t \geq b}|C(t)|\|f\|_{C\left(R_{b}\right)} \cdot \square
\end{aligned}
$$

Lemma 3.3. Let $G\left(x_{0}\right)<\infty$ and $C(x) \rightarrow 0$ as $x \rightarrow \infty$. Then for $x \geq x_{0}$

$$
\int_{x}^{\infty} \frac{\left|K^{j}(C(\cdot) f(\cdot))(t)\right|}{r(t) u_{1}(t) v_{1}(t)} d t \leq\left(\sup _{t \geq x}|C(t)|\right)^{j-1} G(x)\|f\|_{C\left(R_{x}\right)}, j=1,2, \ldots .
$$

Proof. The definition of $K$ implies the inequality for $x \geq x_{0}, j=1,2, \ldots$.

$$
\left|K^{j}(C(\cdot) f(\cdot))(x)\right| \leq K^{j}(|C(\cdot) f(\cdot)|)(x) \leq\left(\sup _{t \geq x}|C(t)|\right)^{j}\|f\|_{C\left(R_{x}\right)} .
$$

Using integration by parts, (3.17), (1.3), (1.4) and (3.5), we obtain:

$$
\begin{aligned}
\int_{x}^{\infty} & \frac{K^{j}(|C(\cdot) f(\cdot)|)(t)}{r(t) u_{1}(t) v_{1}(t)} d t \\
& =\int_{x}^{\infty} \frac{v_{1}(t)}{u_{1}(t)} \frac{1}{r(t) u_{1}(t) v_{1}(t)}\left(\int_{t}^{\infty} \frac{|C(\xi)| K^{j-1}(|C(\cdot) f(\cdot)|)(\xi) d \xi}{r(\xi) v_{1}^{2}(\xi)}\right) d t \\
& =\int_{x}^{\infty}\left(\frac{v_{1}(t)}{u_{1}(t)}\right)^{\prime}\left(\int_{t}^{\infty} \frac{|C(\xi)| K^{j-1}(|C(\cdot) f(\cdot)|)(\xi) d \xi}{r(\xi) v_{1}^{2}(\xi)}\right) d t \\
& =\left.K^{j}(|C(\cdot) f(\cdot)|)(t)\right|_{x} ^{\infty}+\int_{x}^{\infty} \frac{|C(t)| K^{j-1}(|C(\cdot) f(\cdot)|)(t) d t}{r(t) u_{1}(t) v_{1}(t)} \\
& =-K^{j}(|C(\cdot) f(\cdot)|)(x)+\int_{x}^{\infty} \frac{|C(t)| K^{j-1}(|C(\cdot) f(\cdot)|)(t) d t}{r(t) u_{1}(t) v_{1}(t)} \\
& =\int_{x}^{\infty} \frac{|C(t)| K^{j-1}(|C(\cdot) f(\cdot)|)(t)\left[1-\frac{u_{1}(t)}{v_{1}(t)} \cdot \frac{v_{1}(x)}{u_{1}(x)}\right] d t}{r(t) u_{1}(t) v_{1}(t)} \\
& \leq\left(\sup _{t \geq x}|C(t)|\right) \int_{x}^{\infty} \frac{K^{j-1}(|C(\cdot) f(\cdot)|)(t) d t}{r(t) u_{1}(t) v_{1}(t)}
\end{aligned}
$$




$$
\leq \cdots \leq \sup _{t \geq x}|C(t)|^{j-1} \int_{x}^{\infty} \frac{K(|C(\cdot) f(\cdot)|)(t) d t}{r(t) u_{1}(t) v_{1}(t)} .
$$

Let us estimate the integral in the right-hand side of (3.18):

$$
\begin{aligned}
\int_{x}^{\infty} & \frac{K(|C(\cdot) f(\cdot)|)(t) d t}{r(t) u_{1}(t) v_{1}(t)}=\int_{x}^{\infty} \frac{1}{r(t) u_{1}^{2}(t)}\left(\int_{t}^{\infty} \frac{|C(\xi)|^{2}|f(\xi)| d \xi}{r(\xi) v_{1}^{2}(\xi)}\right) d t \\
& =\int_{x}^{\infty}\left(\frac{v_{1}(t)}{u_{1}(t)}\right)^{\prime}\left(\int_{t}^{\infty} \frac{|C(\xi)|^{2}|f(\xi)|}{r(\xi) v_{1}^{2}(\xi)} d \xi\right) d t \\
& =\left.K(|C(\xi) f(\xi)|)(t)\right|_{x} ^{\infty}+\int_{x}^{\infty} \frac{|C(\xi)|^{2}|f(\xi)|}{r(t) u_{1}(t) v_{1}(t)} d t \\
& =\int_{x}^{\infty} \frac{|C(\xi)|^{2}|f(\xi)|}{r(t) u_{1}(t) v_{1}(t)}\left[1-\frac{u_{1}(t)}{v_{1}(t)} \frac{v_{1}(x)}{u_{1}(x)}\right] d t \leq G(x)\|f\|_{C\left(R_{x}\right)} .
\end{aligned}
$$

Inequalities (3.16) follow from the latter inequality, (3.18) and (3.17).

\section{Criterion of SOlvability}

OF THE NARROW HARTMAN-WINTNER PROBLEM

Proof of Theorem 2. Necessity. We divide the proof into separate statements.

Lemma 4.1. If problem $(1.5)-(1 . *)$ is solvable, then $I(x) \in \mathcal{L}\left(x_{0}\right)$.

Proof. By Corollary 2.2, (2.1) - (2.*) is solvable. Let $x_{1}$ be such that $2 \geq|\beta(x)| \geq$ $2^{-1}$ for $x \geq x_{1}$ and $\alpha_{2} \geq \alpha_{1} \geq x_{1}$. From (2.1), integrating by parts we obtain

$$
\begin{aligned}
\int_{\alpha_{1}}^{\alpha_{2}} & \Delta q(t) u_{1}(t) v_{1}(t) d t \\
& =\int_{\alpha_{1}}^{\alpha_{2}}\left(r(t) u_{1}^{2}(t) \beta^{\prime}(t)\right)^{\prime} \frac{v_{1}(t)}{u_{1}(t)} \frac{d t}{\beta(t)} \\
& =\left.\frac{r(t) u_{1}(t) v_{1}(t) \beta^{\prime}(t)}{\beta(t)}\right|_{\alpha_{1}} ^{\alpha_{2}}-\int_{\alpha_{1}}^{\alpha_{2}} \frac{\beta^{\prime}(t)}{\beta(t)} d t+\int_{\alpha_{1}}^{\alpha_{2}} \frac{r(t) u_{1}(t) v_{1}(t) \beta^{\prime}(t)^{2} d t}{\beta^{2}(t)} d t .
\end{aligned}
$$

Then

$$
\begin{aligned}
\left|\int_{\alpha_{1}}^{\alpha_{2}} \Delta q(t) u_{1}(t) v_{1}(t) d t\right| \leq & 2 \sum_{i=1}^{2}\left[r\left(\alpha_{i}\right) u_{1}\left(\alpha_{i}\right) v_{1}\left(\alpha_{i}\right)\left|\beta^{\prime}\left(\alpha_{i}\right)\right|\right] \\
& +\left|\ln \frac{\beta\left(\alpha_{2}\right)}{\beta\left(\alpha_{1}\right)}\right|+4 \int_{\alpha_{1}}^{\alpha_{2}} r(t) u_{1}(t) v_{1}(t)\left|\beta^{\prime}(t)\right|^{2} d t .
\end{aligned}
$$

The statement of the lemma follows from $(2.2),(2 . *)$, the latter inequality and well-known convergency criterion for improper integrals $[6, \S 1.5]$.

Lemma 4.2. If problem $(1.5)-(1 . *)$ is solvable, then $G\left(x_{0}\right)<\infty$.

Proof. According to Lemmas 4.1 and 3.1, we obtain: $C(x) \in \mathcal{L}\left(x_{0}\right), C(x) \rightarrow 0$ as $x \rightarrow \infty$. Let us use Corollaries 2.1 and 2.2. Let $\beta(x)$ be a solution of $(2.8)-(2 . *)$. 
From (2.8), (1.4) and (2.9) we have:

$$
\begin{aligned}
r(x) & u_{1}(x) v_{1}(x) \beta^{\prime}(x) \\
& =-\frac{v_{1}(x)}{u_{1}(x)} \int_{x}^{\infty} \Delta q(t) u_{1}^{2}(t) \beta(t) d t \\
& =\frac{v_{1}(x)}{u_{1}(x)} \int_{x}^{\infty} \beta(t) d\left(\int_{t}^{\infty} \Delta q(\xi) u_{1}^{2}(\xi) d \xi\right) \\
& =\frac{v_{1}(x)}{u_{1}(x)}\left[\left.\beta(t) \int_{t}^{\infty} \Delta q(\xi) u_{1}^{2}(\xi) d \xi\right|_{x} ^{\infty}-\int_{x}^{\infty} \beta^{\prime}(t)\left(\int_{t}^{\infty} \Delta q(\xi) u_{1}^{2}(\xi) d \xi\right) d t\right] \\
& =\frac{v_{1}(x)}{u_{1}(x)}\left[\left.\frac{u_{1}(t)}{v_{1}(t)} C(t) \beta(t)\right|_{x} ^{\infty}-\int_{x}^{\infty} \frac{u_{1}(t)}{v_{1}(t)} C(t) \beta^{\prime}(t) d t\right] \\
& =-C(x) \beta(x)-\frac{v_{1}(x)}{u_{1}(x)} \int_{x}^{\infty} \frac{u_{1}(t)}{v_{1}(t)} C(t) \beta^{\prime}(t) d t .
\end{aligned}
$$

By virtue of (2.9) there exists $x_{1} \geq x_{0}$ such that $|\beta(x)| \geq 2^{-1}$ for $x \geq x_{1}$. Then for $x \geq x_{1}$ from (4.2) and the Schwartz inequality it follows that:

$$
\begin{aligned}
\frac{1}{2}|C(x)| \leq|C(x) \beta(x)| \leq & r(x) u_{1}(x) v_{1}(x)\left|\beta^{\prime}(x)\right| \\
& +\frac{v_{1}(x)}{u_{1}(x)}\left(\int_{x}^{\infty} r(t) u_{1}(t) v_{1}(t)\left|\beta^{\prime}(t)\right|^{2} d t\right)^{1 / 2} \\
& \cdot\left[\int_{x}^{\infty} \frac{1}{r(t) u_{1}(t) v_{1}(t)}\left(\frac{u_{1}(t)}{v_{1}(t)}|C(t)|\right)^{2} d t\right]^{1 / 2} .
\end{aligned}
$$

For the sake of convenience we denote:

$$
\kappa(x)=r(x) u_{1}(x) v_{1}(x)\left|\beta^{\prime}(x)\right|^{2}, \quad S(x)=\int_{x}^{\infty} \kappa(t) d t, \quad x \geq x_{1} .
$$

The previous inequality implies the estimate:

$$
\begin{aligned}
\frac{1}{8}|C(x)|^{2} \leq & r(x) u_{1}(x) v_{1}(x) \kappa(x) \\
& +S(x)\left(\frac{v_{1}(x)}{u_{1}(x)}\right)^{2} \int_{x}^{\infty} \frac{1}{r(t) u_{1}(t) v_{1}(t)}\left(\frac{u_{1}(t)}{v_{1}(t)}|C(t)|\right)^{2} d t .
\end{aligned}
$$

Let us divide this inequality by $r(x) u_{1}(x) v_{1}(x)$ and integrate the result over $[x, x+\theta]$, $\theta>0$ :

$$
\begin{aligned}
& \frac{1}{8} \int_{x}^{x+\theta} \frac{|C(t)|^{2} d t}{r(t) u_{1}(t) v_{1}(t)} \leq S(x)-S(x+\theta) \\
& \quad+\int_{x}^{x+\theta} \frac{S(t)}{r(t) u_{1}^{2}(t)}\left(\frac{v_{1}(t)}{u_{1}(t)}\right)\left[\int_{t}^{\infty} \frac{\left(u_{1}(\xi) v_{1}(\xi)^{-1}|C(\xi)|\right)^{2} d \xi}{r(\xi) u_{1}(\xi) v_{1}(\xi)}\right] d t \\
& \quad \leq S(x)+\frac{S(x)}{2} \int_{x}^{x+\theta}\left[\left(\frac{v_{1}(t)}{u_{1}(t)}\right)^{2}\right]^{\prime}\left[\int_{t}^{\infty} \frac{\left(u_{1}(\xi) v_{1}^{-1}(\xi)|C(\xi)|\right)^{2}}{r(\xi) u_{1}(\xi) v_{1}(\xi)} d \xi\right] d t .
\end{aligned}
$$


We denote the integral in the right hand side of $(4.3)$ by $F(x, \theta)$ and consider it separately. Integrating by parts and using (1.3) and (3.1) we obtain:

$$
\begin{aligned}
F(x, \theta) & =\int_{x}^{x+\theta}\left[\left(\frac{v_{1}(t)}{u_{1}(t)}\right)^{2}\right]^{\prime}\left[\int_{t}^{\infty} \frac{\left(u_{1}(\xi) v_{1}(\xi)^{-1}|C(\xi)|\right)^{2} d \xi}{r(\xi) u_{1}(\xi) v_{1}(\xi)}\right] d t \\
& =\int_{x}^{x+\theta} \frac{|C(t)|^{2} d t}{r(t) u_{1}(t) v_{1}(t)}+\left.\left(\frac{v_{1}(t)}{u_{1}(t)}\right)^{2} \int_{t}^{\infty} \frac{\left(u_{1}(\xi) v_{1}(\xi)-1|C(\xi)|\right)^{2} d \xi}{r(\xi) u_{1}(\xi) v_{1}(\xi)}\right|_{x} ^{x+\theta} \\
& \leq \int_{x}^{x+\theta} \frac{|C(t)|^{2} d t}{r(t) u_{1}(t) v_{1}(t)}+\left(\frac{v_{1}(x+\theta)}{u_{1}(x+\theta)}\right)^{2} \int_{x+\theta}^{\infty} \frac{\left(u_{1}(\xi) v_{1}(\xi)^{-1}|C(\xi)|\right)^{2} d \xi}{r(\xi) u_{1}(\xi) v_{1}(\xi)} \\
& \leq \int_{x}^{x+\theta} \frac{|C(t)|^{2} d t}{r(t) u_{1}(t) v_{1}(t)}+4 A^{2}(x)\left(\frac{v_{1}(x+\theta)}{u_{1}(x+\theta)}\right)^{2} \int_{x+\theta}^{\infty} \frac{1}{r(\xi) v_{1}(\xi)^{2}} \frac{u_{1}(\xi)}{v_{1}(\xi)} d \xi \\
& =\int_{x}^{x+\theta} \frac{|C(t)|^{2} d t}{r(t) u_{1}(t) v_{1}(t)}+4 A^{2}(x)\left(\frac{v_{1}(x+\theta)}{u_{1}(x+\theta)}\right)^{2} \int_{x+\theta}^{\infty}\left[-\frac{1}{2}\left(\frac{u_{1}(\xi)}{v_{1}(\xi)}\right)^{2}\right]^{\prime} d \xi \\
& \leq 2 A^{2}(x)+\int_{x}^{x+\theta} \frac{|C(t)|^{2} d t}{r(t) u_{1}(t) v_{1}(t)} .
\end{aligned}
$$

We substitute this bound into (4.3) and obtain:

$$
\begin{aligned}
\frac{1}{8} \int_{x}^{x+\theta} \frac{|C(t)|^{2} d t}{r(t) u_{1}(t) v_{1}(t)} & \leq S(x)+\frac{S(x)}{2} \cdot F(x, \theta) \leq S(x)+S(x) A^{2}(x) \\
& +\frac{S(x)}{2} \int_{x}^{x+\theta} \frac{|C(t)|^{2} d t}{r(t) u_{1}(t) v_{1}(t)}
\end{aligned}
$$

Therefore,

$$
\frac{1}{8}[1-4 S(x)] \int_{x}^{x+\theta} \frac{|C(t)|^{2} d t}{r(t) u_{1}(t) v_{1}(t)} \leq S(x)\left[1+A^{2}(x)\right] .
$$

Let $x_{1}$ be such that $S(x) \leq 8^{-1}$ for $x \geq x_{1}$. Then

$$
\int_{x}^{x+\theta} \frac{|C(t)|^{2} d t}{r(t) u_{1}(t) v_{1}(t)} \leq 16 S(x)\left(1+A^{2}(x)\right), \quad x \geq x_{1} .
$$

With $\theta$ tending to infinity we obtain $G(x)<\infty$ for $x \geq x_{1}$.

Proof of Theorem 2. Sufficiency. Let $b \geq x_{0}$ be such that $A(b) \leq 4^{-1}$. According to (3.15) and (3.1), we have $\|K\| \leq 2^{-1}$. Therefore, the operator $T=\sum_{i=1}^{\infty}(-1)^{i} K^{i}$ jointly with $K$ acts from $C\left(R_{b}\right)$ to $C\left(R_{b}\right)$ and $\|T\| \leq 1$.

Lemma 4.3. Let $I(x) \in \mathcal{L}\left(x_{0}\right)$ and $A(b) \leq 4^{-1}$. For problems $(2.1)-(2.2)$ and $(2.8)-(2.9)$ to be solvable it is necessary and sufficient that the integral equation

$$
\gamma(x)=\exp (H(x))+\int_{x}^{\infty} \exp (H(x)-H(t)) \frac{T(C(\cdot) \gamma(\cdot))(t)}{r(t) u_{1}(t) v_{1}(t)} d t, \quad x \geq b,
$$

has a solution $\gamma(x) \in C\left(R_{b}\right)$. In the latter case $\gamma(x)$ coincides with the solution $\beta(x)$ of problems $(2.1)-(2.2),(2.8)-(2.9)$.

Proof. Necessity. Let $I(x) \in \mathcal{L}\left(x_{0}\right)$ and let $\beta(x)$ be a solution of $(2.8)-(2.9)$. Then (4.2) holds. Denote $\mu(x)=r(x) u_{1}(x) v_{1}(x) \beta^{\prime}(x)$ and write (4.2) in terms of 
operator $K$ :

$$
\mu(x)=-C(x) \beta(x)-(K \mu)(x), \quad x \geq b .
$$

Let $E$ be the identity operator in $C\left(R_{b}\right)$. Since $\|K\| \leq 2^{-1}$, then $(E+K)$ is invertible in $C\left(R_{b}\right)$. Hence, solving (4.4) with respect to $\mu(x)$, we obtain:

$$
\begin{aligned}
\mu(x) & =r(x) u_{1}(x) v_{1}(x) \beta^{\prime}(x)=-(E+K)^{-1}(C(\cdot) \beta(\cdot))(x) \\
& =-C(x) \beta(x)-T(C(\cdot) \beta(\cdot))(x) .
\end{aligned}
$$

According to Lemma 3.1, $H(x)$ converges and hence for $x \geq b(4.5)$ implies

$$
\begin{aligned}
\beta^{\prime}(x)+\frac{C(x) \beta(x)}{r(x) u_{1}(x) v_{1}(x)} & \equiv \exp (H(x)) \frac{d}{d x}[\exp (-H(x)) \beta(x)] \\
& =-\frac{T(C(\cdot) \beta(\cdot))(x)}{r(x) u_{1}(x) v_{1}(x)}
\end{aligned}
$$

Now (4.4) with $\gamma(x)=\beta(x)$ follows from (4.7) and (2.9).

Sufficiency. Let $\gamma(x)$ be a solution of (4.4) and $\gamma(x) \in C\left(R_{b}\right)$. Then, obviously, $\gamma(x) \rightarrow 1$ as $x \rightarrow \infty$. From (4.4) we find

$$
\begin{aligned}
r(x) u_{1}(x) v_{1}(x) \gamma^{\prime}(x) & =-C(x) \gamma(x)-T(C(\cdot) \gamma(\cdot))(x) \\
& =-(E+K)^{-1}(C(\cdot) \gamma(\cdot))(x),
\end{aligned}
$$

and from (4.8), Lemma 3.1 and (3.15) we obtain:

$$
r(x) u_{1}(x) v_{1}(x)\left|\gamma^{\prime}(x)\right| \leq \tau\|C(\cdot) \gamma(\cdot)\|_{C\left(R_{x}\right)} \leq \tau A(x)\|\gamma\|_{C\left(R_{b}\right)} .
$$

Therefore, $r(x) u_{1}(x) v_{1}(x) \gamma^{\prime}(x) \rightarrow 0$ as $x \rightarrow \infty$. Furthermore, (4.8) and (3.7) yield:

$$
\begin{aligned}
& r(x) u_{1}(x) v_{1}(x) \gamma^{\prime}(x)=-C(x) \gamma(x)-K\left(r(\cdot) u_{1}(\cdot) v_{1}(\cdot) \gamma^{\prime}(\cdot)\right)(x) \\
& =-C(x) \gamma(x)-\frac{v_{1}(x)}{u_{1}(x)} \int_{x}^{\infty} C(t) \frac{u_{1}(t)}{v_{1}(t)} \gamma^{\prime}(t) d t \\
& =-\gamma(x) C(x)-\frac{v_{1}(x)}{u_{1}(x)}\left\{\left.\gamma(t) C(t) \frac{u_{1}(t)}{v_{1}(t)}\right|_{x} ^{\infty}\right. \\
& \left.\quad-\int_{x}^{\infty} \gamma(t)\left[C^{\prime}(t) \frac{u_{1}(t)}{v_{1}(t)}-\frac{C(t)}{r(t) v_{1}^{2}(t)}\right] d t\right\} \\
& =-C(x) \gamma(x)+C(x) \gamma(x)+\frac{v_{1}(x)}{u_{1}(x)} \int_{x}^{\infty} \gamma(t) \frac{u_{1}(t)}{v_{1}(t)}\left[C^{\prime}(t)-\frac{C(t)}{r(t) u_{1}(t) v_{1}(t)}\right] d t \\
& =-\frac{v_{1}(x)}{u_{1}(x)} \int_{x}^{\infty} \Delta q(t) u_{1}^{2}(t) \gamma(t) d t .
\end{aligned}
$$

Thus problem (2.8) - (2.9) is solvable and $\beta(x)=\gamma(x)$.

Let us check now that the conditions of Theorem 2 are sufficient for the solvability of problem $(1.5)-(1 . *)$. Let us consider the operator

$$
(Q \gamma)(x)=\int_{x}^{\infty} \exp (H(t)-H(x)) \frac{T(C(\cdot) \gamma(\cdot))(t) d t}{r(t) u_{1}(t) v_{1}(t)}, \gamma(x) \in C\left(R_{b}\right), b \gg 1
$$

By virtue of Lemmas 3.1, 3.3 and the well-known theorem on term by term integration of series $([6, \S 10.9])$, the operator $Q$ maps $C\left(R_{b}\right)$ into $C\left(R_{b}\right)$ and

$$
\|Q\| \leq \tau G(b)=\tau \int_{b}^{\infty} \frac{|C(t)|^{2} d t}{r(t) u_{1}(t) v_{1}(t)} .
$$


Increasing, if necessary, the number $b$ we obtain $\|Q\| \leq 2^{-1}$. Hence $Q$ is a contracting mapping in $C\left(R_{b}\right)$ and (4.3) has solution $\gamma(x) \in C\left(R_{b}\right)$. Then, according to Lemmas 4.3 and 2.1 , the problem $(1.5)-(1.7)$ is solvable.

Now we will need the statement:

Lemma 4.4. Let $C(x) \in \mathcal{L}\left(x_{0}\right), \lim _{x \rightarrow \infty} C(x)=0$ and let problem $(1.5)-(1.7)$ be solvable. If $G\left(x_{0}\right)<\infty$, then $(1.5)-(1.7)$ imply (1.*).

Proof. According to the conditions of the lemma we can repeat the derivation of (4.2) from Lemma 4.2. Using (2.9) and the Schwartz inequality, we find:

$$
\begin{aligned}
r(x) & u_{1}(x) v_{1}(x)\left|\beta^{\prime}(x)\right| \leq|C(x)||\beta(x)|+\frac{v_{1}(x)}{u_{1}(x)} \int_{x}^{\infty}|C(t)| \frac{u_{1}(x)}{v_{1}(x)}\left|\beta^{\prime}(t)\right| d t \\
& \leq|C(x)||\beta(x)|+\left(\sup _{t \geq x}\left(r(t) u_{1}(t) v_{1}(t)\left|\beta^{\prime}(t)\right|\right)\right) \frac{v_{1}(x)}{u_{1}(x)} \int_{x}^{\infty} \frac{|C(t)| d t}{r(t) v_{1}^{2}(t)} \\
& \leq \tau\left\{|C(x)|+\frac{v_{1}(x)}{u_{1}(x)} \int_{x}^{\infty} \frac{|C(t)| d t}{r(t) v_{1}^{2}(t)}\right\} \\
& \leq \tau\left\{|C(x)|+\frac{v_{1}(x)}{u_{1}(x)}\left[\int_{x}^{\infty} \frac{|C(t)|^{2} d t}{r(t) v_{1}^{2}(t)}\right]^{1 / 2}\left[\int_{x}^{\infty} \frac{d t}{r(t) v_{1}^{2}(t)}\right]^{2}\right\} \\
& =\tau\left\{|C(x)|+\left[\frac{v_{1}(x)}{u_{1}(x)} \int_{x}^{\infty} \frac{|C(t)|^{2} d t}{r(t) v_{1}^{2}(t)}\right]^{1 / 2}\right\} .
\end{aligned}
$$

It follows from the latter inequality that

$$
\begin{gathered}
r(x) u_{1}(x) v_{1}(x)\left|\beta^{\prime}(x)\right|^{2} \leq \tau\left\{\frac{|C(x)|^{2}}{r(x) u_{1}(x) v_{1}(x)}+\frac{1}{r(x) u_{1}^{2}(x)} \int_{x}^{\infty} \frac{|C(t)|^{2} d t}{r(t) v_{1}^{2}(t)}\right\} \\
=\tau\left\{\frac{|C(x)|^{2}}{r(x) u_{1}(x) v_{1}(x)}+\left(\frac{v_{1}(x)}{u_{1}(x)}\right)^{\prime} \int_{x}^{\infty} \frac{|C(t)|^{2} d t}{r(t) v_{1}^{2}(t)}\right\} .
\end{gathered}
$$

Let us integrate this estimate over $[x, \infty)$ and use $(3.5)$ :

$$
\begin{aligned}
\int_{x}^{\infty} r(t) u_{1}(t) v_{1}(t)\left|\beta^{\prime}(t)\right|^{2} d t \\
\leq \tau\left\{G(x)+\int_{x}^{\infty}\left(\frac{v_{1}(t)}{u_{1}(t)}\right)^{\prime}\left(\int_{t}^{\infty} \frac{|C(\xi)|^{2} d \xi}{r(\xi) v_{1}^{2}(\xi)}\right) d t\right\} \\
=\tau\left\{G(x)+\left.\frac{v_{1}(t)}{u_{1}(t)} \int_{t}^{\infty} \frac{|C(\xi)|^{2} d \xi}{r(\xi) v_{1}^{2}(\xi)}\right|_{x} ^{\infty}+\int_{x}^{\infty} \frac{|C(t)|^{2} d t}{r(t) u_{1}(t) v_{1}(t)}\right\} \\
\leq \tau\left\{2 G(x)+\lim _{t \rightarrow \infty} \frac{v_{1}(t)}{u_{1}(t)} \int_{t}^{\infty} \frac{|C(\xi)|^{2} d \xi}{r(\xi) v_{1}^{2}(\xi)}\right\} \\
\leq \tau\left\{G(x)+\lim _{t \rightarrow \infty} \sup _{\xi \geq t}|C(\xi)|^{2}\right\}=\tau G(x) .
\end{aligned}
$$

The proof of Theorem 2 follows from Lemma 4.4. 
Proof of Corollary of Theorem 2. If (I) is fulfilled, then $P(x) \in \mathcal{L}\left(x_{0}\right)$, and by Lemma $3.1 G\left(x_{0}\right)<\infty$. It remains to refer to Theorem 2. In particular, if $I(x)$ converges absolutely, then (I) holds automatically. Similarly, if (II) is fulfilled, then $J(x) \in \mathcal{L}\left(x_{0}\right)$ and by $(3.2), G\left(x_{0}\right)<\infty$. In particular, according to (3.1) we have:

$$
\int_{x_{0}}^{\infty} \frac{|I(t) C(t)| d t}{r(t) u_{1}(t) v_{1}(t)} \leq 2 \min \left\{\int_{x_{0}}^{\infty} \frac{A(t)|C(t)| d t}{r(t) u_{1}(t) v_{1}(t)} ; \int_{x_{0}}^{\infty} \frac{|I(t)| A(t)}{r(t) u_{1}(t) v_{1}(t)} d t\right\}
$$

and if the right-hand side of (4.10) is finite, we obtain $J(x) \in \mathcal{L}\left(x_{0}\right)$ and hence $G\left(x_{0}\right)<\infty$. Case (III) easily follows from Lemma 3.1 and Theorem 2 .

Proof of Corollary 2.3. We have to check only one implication: if $I(x) \in \mathcal{L}\left(x_{0}\right)$, $\operatorname{Im} q(x) \equiv 0$ and problem (1.5) - (1.7) is solvable, then (1.*) is fulfilled. As shown in section 2, problems (1.5) - (1.7) and (2.1) - (2.2) are equivalent, and condition (1.*) for problem $(1.5)-(1.7)$ is equivalent to condition $(2 . *)$ for problem $(2.1)$ - (2.2). Therefore, to prove the lemma it is sufficient to establish that if $I(x) \in$ $\mathcal{L}\left(x_{0}\right), \operatorname{Im} q(x) \equiv 0$ and problem $(2.1)-(2.2)$ is solvable, then $(2 . *)$ is fulfilled. Let $I(x) \in \mathcal{L}\left(x_{0}\right), \operatorname{Im} q(x) \equiv 0$ and $\beta(x)$ be a solution of $(2.1)-(2.2)$. We set $\alpha_{1}=x_{1}$, $\alpha_{2}=\infty$ in formula (4.1) (see notations of Lemma 4.1). Then

$$
\int_{x_{1}}^{\infty} r(t) u_{1}(t) v_{1}(t) \frac{\beta^{\prime}(t)^{2}}{\beta^{2}(t)}=I\left(x_{1}\right)+\frac{r\left(x_{1}\right) u_{1}\left(x_{1}\right) \beta^{\prime}\left(x_{1}\right)}{\beta\left(x_{1}\right)}-\ln \beta\left(x_{1}\right) .
$$

Since $\beta^{\prime}(t)$ is a real function, and $\beta(t) \leq 2$ for $t \geq x_{1}$, then

$$
\int_{x_{1}}^{\infty} r(t) u_{1}(t) v_{1}(t)\left|\beta^{\prime}(t)\right|^{2} d t \leq 4\left\{\left|I\left(x_{1}\right)\right|+r\left(x_{1}\right) v_{1}\left(x_{1}\right) u_{1}\left(x_{1}\right)\left|\beta^{\prime}\left(x_{1}\right)\right|+\left|\ln \beta\left(x_{1}\right)\right|\right\} .
$$

\section{Relation Between the Hartman-Wintner Problem AND ITS CONTRACTION}

In this section we prove Theorem 3. Let problem (1.5) - (1.7) be solvable. Then there exists a solution $\beta(x)$ of problem (2.1) - (2.2) and a number $x_{1}$ such that $|\beta(x)|>2^{-1}$ for $x \geq x_{1}$. Using (2.1), (2.2) and (1.4) for $x \geq x_{1}$ we obtain:

$$
\begin{aligned}
\int_{x}^{\infty} & \Delta q(t) u_{1}^{2}(t) d t=\int_{x}^{\infty} \frac{\left(r(t) u_{1}^{2}(t) \beta^{\prime}(t)\right)^{\prime} d t}{\beta(t)} \\
& =\left.\frac{r(t) u_{1}^{2}(t) \beta^{\prime}(t)}{\beta(t)}\right|_{x} ^{\infty}+\int_{x}^{\infty} \frac{r(t) u_{1}^{2}(t) \beta^{\prime 2}(t) d t}{\beta^{2}(t)} \\
& =\left(\left.r(t) u_{1}(t) v_{1}(t) \beta^{\prime}(t) \frac{u_{1}(t)}{v_{1}(t)} \frac{1}{\beta(t)}\right|_{x} ^{\infty}+\int_{x}^{\infty} \frac{\left(r(t) u_{1}(t) v_{1}(t) \beta^{\prime}(t)\right)^{2} d t}{r(t) v_{1}^{2}(t)}\right. \\
& =-r(x) u_{1}^{2}(x) \beta^{\prime}(x) \frac{1}{\beta(x)}+\int_{x}^{\infty} \frac{\left(r(t) u_{1}(t) v_{1}(t) \beta^{\prime}(t)\right)^{2} d t}{r(t) v_{1}^{2}(t)} .
\end{aligned}
$$


It follows from the latter equality, (1.4) and (2.2) that the integral in the left hand side converges. Therefore, $C(x)$ exists, and for $x \geq x_{1}$ we obtain:

$$
\begin{aligned}
|C(x)|= & \left|\frac{v_{1}(x)}{u_{1}(x)} \int_{x}^{\infty} \Delta q(t) u_{1}^{2}(t) d t\right| \\
\leq & \frac{r(x) u_{1}(x) v_{1}(x)\left|\beta^{\prime}(x)\right|}{|\beta(x)|}+\frac{v_{1}(x)}{u_{1}(x)} \int_{x}^{\infty} \frac{\left(r(t) u_{1}(t) v_{1}(t)\left|\beta^{\prime}(t)\right|\right)^{2} d t}{r(t) v_{1}^{2}(t)} \\
\leq & 2 \sup _{t \geq x}\left(r(t) u_{1}(t) v_{1}(t)\left|\beta^{\prime}(t)\right|\right) \\
& \cdot\left\{1+\sup _{t \geq x} r(t) u_{1}(t) v_{1}(t)\left|\beta^{\prime}(t)\right| \frac{1}{u_{1}(x)} v_{1}(x) \int_{x}^{\infty} \frac{d t}{r(t) v_{1}^{2}(t)}\right\} \\
\leq & \tau \sup _{t \geq x} r(t) u_{1}(t) v_{1}(t)\left|\beta^{\prime}(t)\right| .
\end{aligned}
$$

Hence, $C(x) \rightarrow 0$ as $x \rightarrow \infty$. Futhermore, if problem (1.5) - (1.7) is solvable and $G\left(x_{0}\right)<\infty$, then, according to Lemma 4.4, $(2 . *)$ is fulfilled and by virtue of Corollary 2.2 relation $(1 . *)$ is fulfilled as well. Conversely, let (1.*) follow from $(1.5)-(1.7)$. Then problem $(1.5)-(1 . *)$ is solvable, and according to Theorem 2 we obtain $G\left(x_{0}\right)<\infty$.

We wish to thank the referee whose valuable remarks and suggestions led to an essential improvement in this paper.

\section{REFERENCES}

1. S. Chen, Asymptotic integrations of nonoscillatory second order differential equations, Trans. Amer. Math. Soc. 327 (2) (1991). MR 92a:34057

2. N. Chernyavskaya and L. Shuster, Asymptotic integration of a nonoscillatory second order differential equation with a linear perturbation, AMS PPS \# 199508-34-001, preprint.

3. P. Hartman, Ordinary Differential Equations, Wiley, New York, 1964. MR 30:1270

4. P. Hartman and A. Wintner, On non-oscillatory linear equations, Amer. J. Math. 75 (1953), 717-730. MR 15:527c

5. J. Simśa, Asymptotic integration of a second order ordinary differential equation, Proc. Amer. Math. Soc. 101 (1) (1987), 96-100. MR 89b:34129

6. C.C. Titchmarsh, The Theory of Functions, Oxford, 1932.

7. W.F. Trench, Linear perturbations of a nonoscillatory second order equation, Proc. Amer. Math. Soc. 97 (3) (1986), 423-428. MR 87g:34036

(N. Chernyavskaya) Department of Mathematics and Computer Science, Ben-Gurion University of the Negev, P.O.B. 653, Beer-Sheva, 84105, IsRael

E-mail address: nina@math.bgu.ac.il

(N. Chernyavskaya) Department of Agricultural Economics and Management, Hebrew University of Jerusalem, P.O.B. 12, Rehovot 76100, Israel

(L. Shuster) Department of Mathematics and Computer Science, Bar-Ilan University, RAMAT-Gan, 52900, IsRAEL 